\title{
Rethinking the Triggering Inflammatory Processes of Chronic Periaortitis
}

\author{
Pascal Meier ${ }^{\mathrm{a}, \mathrm{b}}$ Bruno Vogt ${ }^{\mathrm{a}}$ Edouard Blanc $^{\mathrm{b}}$ \\ aService of Nephrology, Department of Medicine, Centre Hospitalier Universitaire Vaudois, Lausanne, and \\ ${ }^{b}$ Division of Nephrology, Department of Medicine, Hôpital de Sion, Sion, Switzerland
}

\section{Key Words}

Chronic periaortitis - Idiopathic retroperitoneal fibrosis • Inflammatory abdominal aortic aneurysm - Perianeurysmal retroperitoneal fibrosis · Atherosclerosis · Inflammation

\begin{abstract}
Chronic periaortitis (CP) is an uncommon inflammatory disease which primarily involves the infrarenal portion of the abdominal aorta. However, $\mathrm{CP}$ should be regarded as a generalized disease with three different pathophysiological entities, namely idiopathic retroperitoneal fibrosis (RPF), inflammatory abdominal aortic aneurysm and perianeurysmal RPF. These entities share similar histopathological characteristics and finally will lead to fibrosis of the retroperitoneal space. Beside fibrosis, an infiltrate with variable chronic inflammatory cell is present. The majority of these cells are lymphocytes and macrophages as well as vascular endothelial cells, most of which are HLA-DR-positive. B and $T$ cells are present with a majority of $T$ cells of the T-helper phenotype. Cytokine gene expression analysis shows the presence of interleukin (IL)- $1 \alpha$, IL-2, IL-4, interferon- $\gamma$ and IL-2 receptors. Adhesion molecules such as E-selectin, intercellular adhesion molecule- 1 and the vascular cell adhesion molecule- 1 were also found in aortic tissue, and may play a significant role in CP pathophysiology. Although CP pathogenesis remains unknown, an exaggerated inflammatory
\end{abstract}

response to advanced atherosclerosis (ATS) has been postulated to be the main process. Autoimmunity has also been proposed as a contributing factor based on immunohistochemical studies. The suspected allergen may be a component of ceroid, which is elaborated within the atheroma. We review the pathogenesis and the pathophysiology of CP, and its potential links with ATS. Clinically relevant issues are summarized in each section with regard to the current working hypothesis of this complex inflammatory disease.

Copyright $\odot 2007$ S. Karger AG, Basel

\section{Introduction}

The term chronic periaortitis (CP) refers to a family of inflammatory diseases with similar morphologic appearance, namely idiopathic retroperitoneal fibrosis (RPF), inflammatory abdominal aorticaneurysm (IAAA) and perianeurysmal RPF $[1,2]$. A pathogenetic role of atherosclerosis (ATS) in the development of CP has been suggested and supported by several previous observations $[3,4]$. In the entities included in CP, except for the degree of aortic dilatation, the histopathological findings appear similar between idiopathic RPF and IAAA [5]. In idiopathic RPF, the aorta is not dilated and the surrounding fibroinflammatory tissue might or might not encase

\section{KARGER}

Fax +4161306 1234

E-Mail karger@karger.ch

www.karger.com
(C) 2007 S. Karger AG, Basel

$1660-2129 / 07 / 1051-0017 \$ 23.50 / 0$

Accessible online at:

www.karger.com/nee
Pascal Meier, MD, FASN

Service of Nephrology/Hypertension

Centre Hospitalier Universitaire Vaudois (CHUV)

Rue du Bugnon, CH-1011 Lausanne (Switzerland)

Tel. +41 21314 1114, Fax +41 21314 1139, E-Mail Pascal.Meier@chuv.ch 
adjacent structures; in IAAA, the tissue develops around a dilated aorta, but does not cause obstructions, and perianeurysmal RPF involves an inflammatory aneurysm, the surrounding tissue of which entraps the adjacent organs (fig. 1) [2, 6].

The clinical presentation of $\mathrm{CP}$ may be different according to the anatomic location. When the ureter is involved, pain in the low back region is common, and may be associated with low-grade fever and weight loss. The evolution is characterized by an obstructive uropathy which may remain asymptomatic until it leads to renal insufficiency. Arterial invasion with aortic aneurysm, or portal hypertension and/or bile duct obstruction as well as extrinsic venous compression with deep vein thrombosis of the legs may also occur. Computed tomography or magnetic resonance represents the best imagery technique to diagnose and manage CP. Multiple deep biopsies are often required to ascertain this diagnosis, and surgical treatment may sometimes be necessary to relieve ureteral obstruction or to cure aneurysmal aorta. Apart from corticosteroids, which are the most used drugs, immunosuppressants are also frequently used. Among these, azathioprine, methotrexate, cyclosporine, and mycophenolate mofetil can induce stable disease remissions and regression of the mass. Finally, tamoxifen has been used successfully in several cases. The outlook for patients is usually good, but severe complications such as chronic renal failure can arise.

\section{Pathophysiology}

The pathogenesis of $\mathrm{CP}$ is only partially known, and the role of ATS in CP remains controversial, mainly because no substantial difference in the incidence of advanced atherosclerotic disease has been clearly demonstrated between CP patients and healthy controls. The leading theory for the pathogenesis of $\mathrm{CP}$ was proposed by Mitchinson [3] and Parums [6] who first suggested that $\mathrm{CP}$ could be a consequence of a local inflammatory reaction to oxidized low-density lipoproteins (oxLDL) and ceroid, which are often found in the atherosclerotic plaques of the abdominal aorta. This hypothesis was supported by an observation of Martina et al. [7] in patients suffering from idiopathic RPF associated with a high incidence of aortic ATS. However, these results are not uniform and progression after open or endovascular repair have been reported $[8,9]$.

On the basis of the works of Mitchinson and Parums, Vaglio et al. [2] recently proposed two potential CP patho- genetic mechanisms: autoallergic aortitis and vasa vasorum vasculitis (fig. 2).

In contrast with atherosclerotic plaques where the intimal layer is mainly affected, abdominal aortic aneurysms are characterized by the formation of inflammatory infiltrates, predominantly in the media and the adventitia. At the early stage, many lymphocytes are seen, and germinal centers are common. The majority of lymphocytes, macrophages and most vascular endothelial cells are HLA-DR-positive. Ki67 and BerH2 staining is found in B cells and T-helper cells, indicating that these cells were proliferating and activated. The characterization of inflammatory cells found in IAAA and in atherosclerotic arteries represents the spectrum of inflammation seen as a complication of advanced ATS.

The inflammatory process of the aortic vasa vasorum and periaortic retroperitoneal vessels may lead to the development of $\mathrm{CP}$, by promoting ATS and aneurysm formation. Its extension into the adjacent retroperitoneum could give rise to the fibroinflammatory reaction typical of CP. Vasa vasorum capillaries may facilitate leukocyte entry into lesions, perfuse the vessel wall beyond diffusion limits from the artery lumen, or cause intraplaque hemorrhage [10]. Plaque neovascularization correlates with lesion size, and may function as conduits for the entry of leukocytes and plasma components into the artery wall. The endothelium of intimal capillaries shows increased E-selectin, intercellular adhesion molecule-1 (ICAM-1), vascular cell adhesion molecule-1 (VCAM-1) and monocyte chemoattractant protein-1 levels compared with the artery surface [10]. Recent work on adhesion molecules and human ATS showed that as aortic ATS develops, ICAM-1 expression is associated with intimal lymphocyte and macrophage populations, and that E-selectin and VCAM-1 expression in the aortic adventitia increases with the severity of the atheromatous plaque as well as the development of adventitial inflammatory cell infiltrates [11]. Ramshaw et al. [12] have shown that ICAM- 1 and especially VCAM- 1 correlated strongly with the adventitial mononuclear cell infiltrate, suggesting its importance in the development of this B-cell-dominant chronic inflammatory response.

Structural alterations of the aortic wall seen in abdominal aortic aneurysms result in part from degradation of the macromolecules, collagen and elastin. These changes are associated with excessive production of matrix metalloproteinases (MMPs) [13], which are assumed to orchestrate the widespread matrix destruction. The inflammatory infiltrate is thought to play an etiologic role in aneurysm formation by direct local production of ma- 
Fig. 1. Schematic representation of idiopathic retroperitoneal fibrosis (RPF), inflammatory abdominal aortic aneurysm (IAAA), and perianeurysmal RPF. In idiopathic RPF, the aorta is undilated with a periaortic fibrosis cuff, which may entrap the adjacent retroperitoneal structures such as the ureters. In IAAA, the inflamed aorta is dilated without periaortic fibrosis. In perianeurysmal RPF, the inflamed aorta is dilated and is responsible for a periaortic fibrosis, which may as in idiopathic RPF, entrap adjacent retroperitoneal structures. Reprinted from Vaglio et al. [2], with permission.

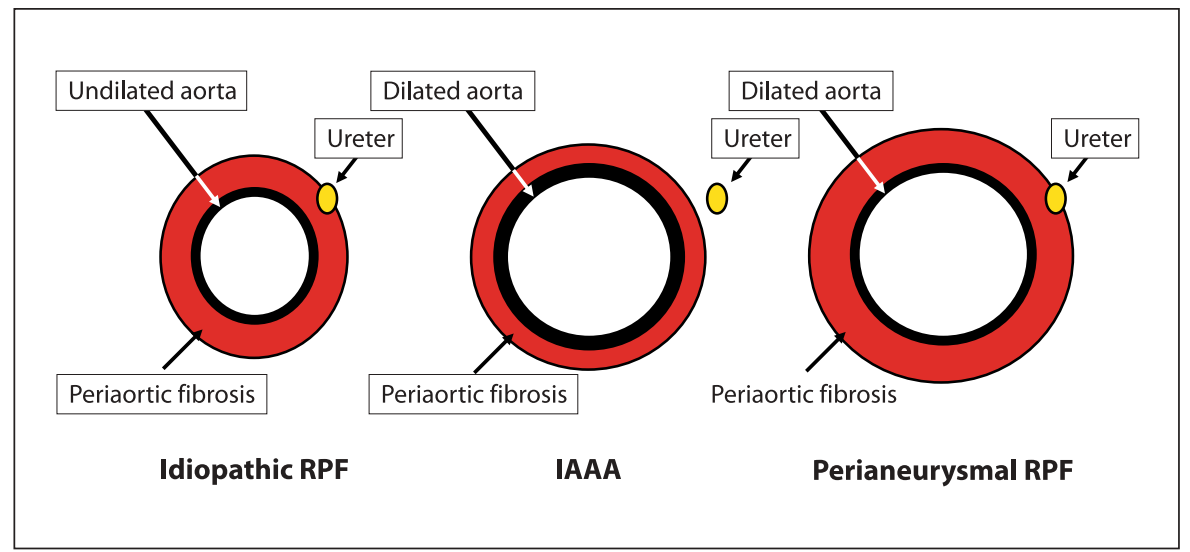

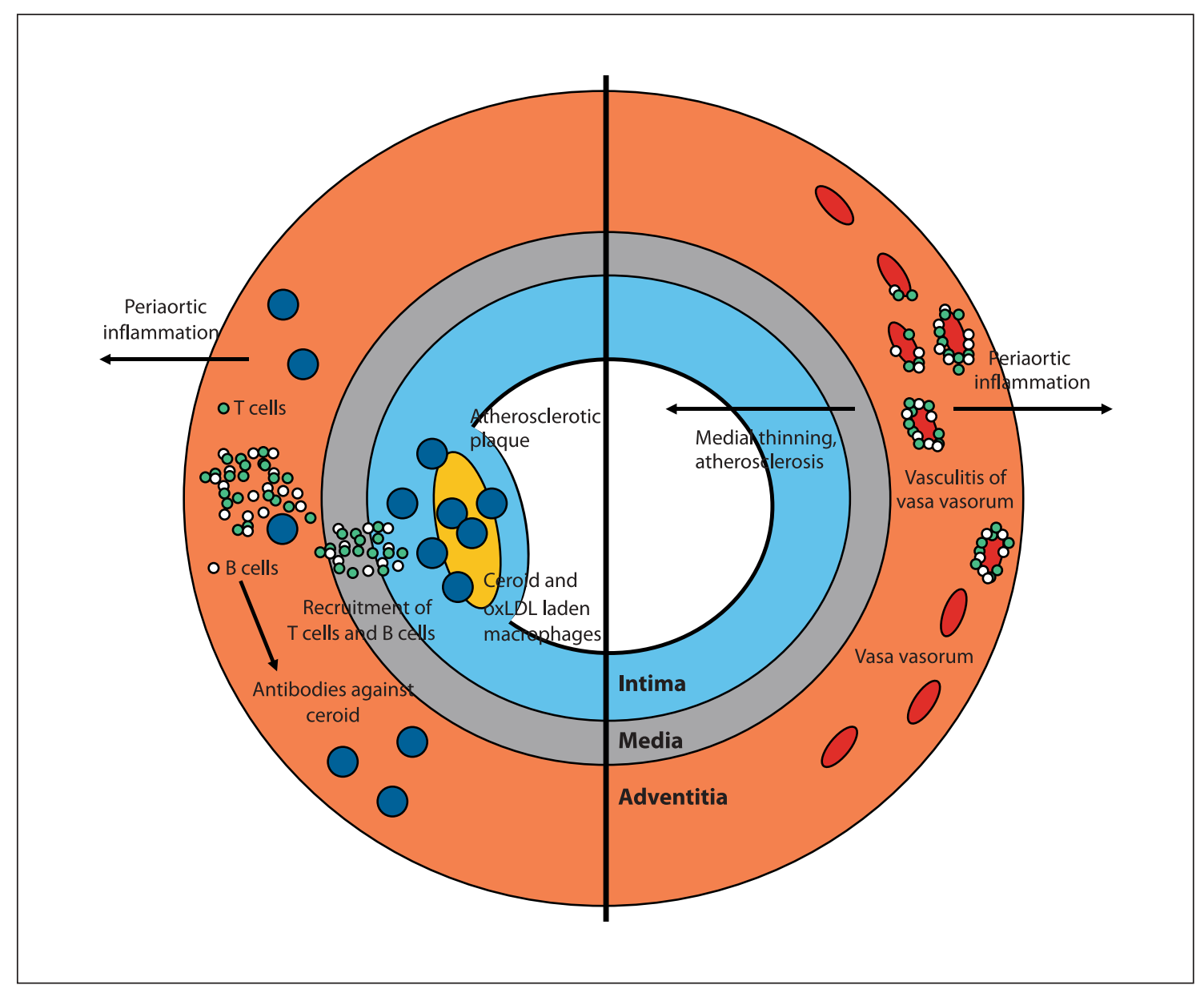

Fig. 2. Two potential pathophysiological mechanisms of chronic periaortitis. The hypothesis of autoallergic aortitis is presented on the left-hand side. In the atherosclerotic plaque, macrophages elaborate antigens, such as oxidized LDL (oxLDL) and ceroid, and present them to $\mathrm{B}$ cells and $\mathrm{T}$ cells. These are recruited and activated in medial and adventitial aortic layers. B cells produce antibodies to ceroid, which are found in close apposition to extracellular ceroid. The inflammatory reaction progress then to the peri- aortic retroperitoneal space. On the right-hand side, chronic periaortitis is initiated in adventitia, with an inflammatory involvement of vasa vasorum (vasa vasorum vasculitis). This inflammatory process can cause weakening of aortic wall with medial thinning and promote atherosclerosis. This phenomenon may extend into surrounding retroperitoneal space. Reprinted from Vaglio et al. [2], with permission. 


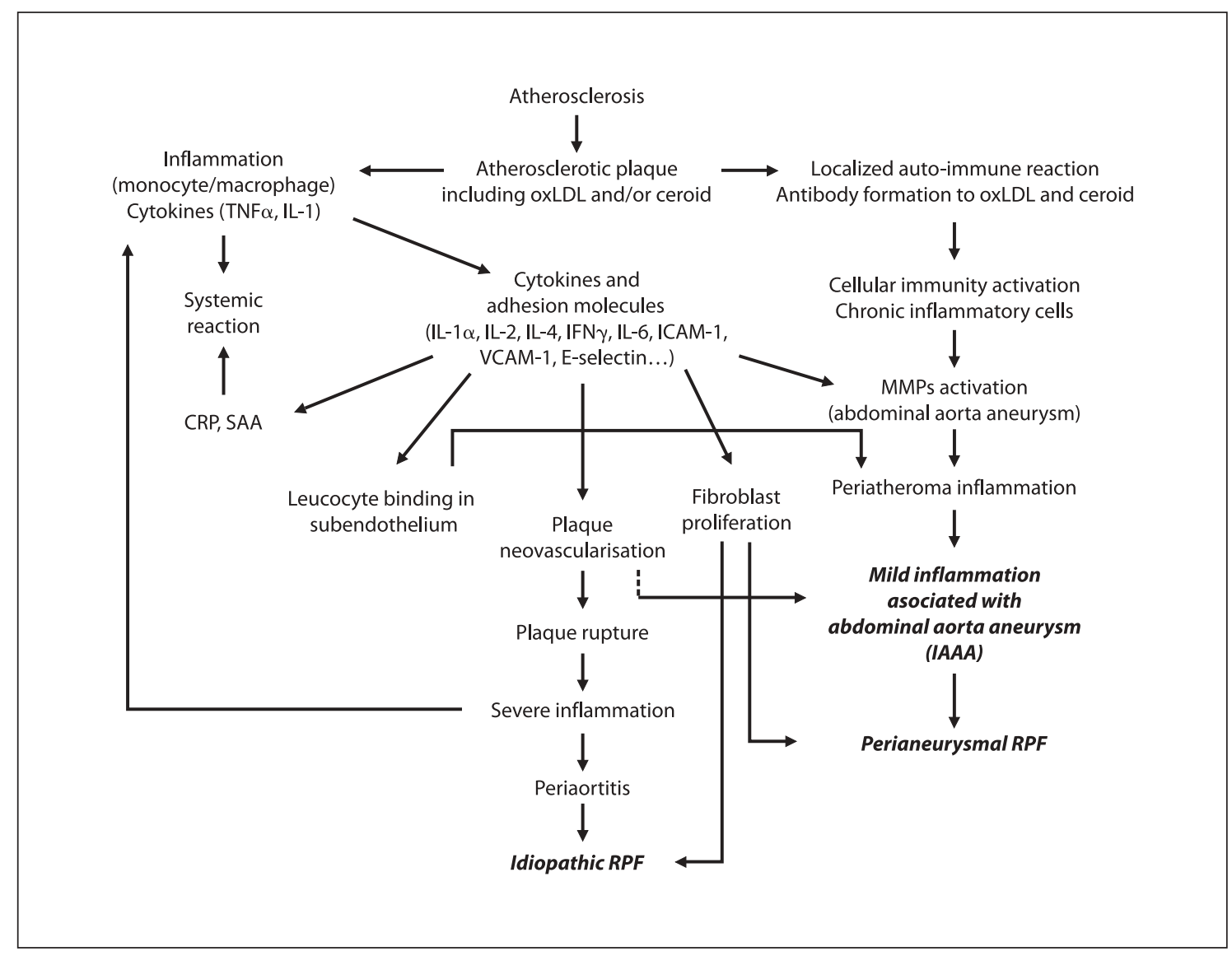

Fig. 3. Potential link between atherosclerosis and the development of chronic periaortitis. Names in italic represent the three entities referred to chronic periaortitis. oxLDL: Oxidized low-density lipoproteins; TNF $\alpha$ : tumor necrosing factor- $\alpha$; IL: interleukin; IFN $\gamma$ : interferon- $\gamma$; ICAM-1: intercellular adhesion molecule-1; VCAM-1: vascular cell adhesion molecule-1; CRP: C-reactive protein; SAA: serum amyloid A; MMPs: metalloproteinases; RPF: retroperitoneal fibrosis.

trix-degrading enzymes and production of cytokines that induce resident mesenchymal cell production of MMPs [14]. Recent findings suggest that both the local mesenchymal cell expression and the macrophage expression of MMPs are required for aneurysm formation [15] (fig. 3). Both fibrillar collagen and elastin are highly organized in the lamellar structure of the aortic media. One potential mechanism for the complementary role of MMP-2 and MMP-9 is that MMP-2 primarily acts as a collagenase-initiating cleavage of the triple helix into one- and three-quarter lengths. The single $\alpha$ chains could then be degraded by MMP-9, releasing the coiled elastin and causing it to become fattened and attenuated. Rupture and expansion rates of abdominal aortic aneurysms have been linked to MMP-2 and MMP-9 levels in tissue and plasma [16]. Such observations appear consistent with the increased medial atrophy observed within abdominal aortic aneurysms, because activated MMPs may weaken the media by causing destruction of elastic and collagen fibers and smooth muscle cells.

\section{Potential Role of Atherosclerosis in Chronic Periaortitis}

The concept that ATS is a specific form of chronic inflammatory process resulting from interactions between plasma lipoproteins, cellular components (monocyte/ macrophages, $\mathrm{T}$ lymphocytes, endothelial cells and smooth muscle cells) and the extracellular matrix of the arterial wall is now well accepted. Histologically, atherosclerotic lesions from the early stage (fatty streak) to more 
complicated lesions possess all the features of chronic inflammation. It has been demonstrated that atherogenic lipoproteins such as oxLDL, remnant lipoprotein $(\beta$-VLDL) and lipoprotein-a play a critical role in the proinflammatory reaction, whereas high-density lipoprotein, anti-atherogenic lipoproteins, exert anti-inflammatory functions. In cholesterol-fed animals, the earliest events in the arterial wall during atherogenesis are the adhesion of monocytes and lymphocytes to endothelial cells followed by the migration of these cells into the intima. These early events in ATS are triggered by the presence of high levels of atherogenic lipoproteins in the plasma and are mediated by inflammatory factors such as adhesion molecules and cytokines in the arterial wall. The development of genetically modified laboratory animals has provided a powerful approach for dissecting individual candidate genes and studying their cause-andeffect relationships in lesion formation and progression. It appears then that $\mathrm{CP}$ is caused by an autoallergic reaction to oxLDL formed in the developing atheromatous plaque and which is normally sequestered unless the media is breached. Serum antibodies (mostly IgG) to oxLDL or ceroid (usually both) will be produced when the aortic media surrounding the atherosclerotic plaques is breached so that the antigen can be recognized by lymphocytes $[1,3,6]$. This proposed cause can explain some observations in CP. For instance, the occasional association of $\mathrm{CP}$ with mediastinal fibrosis is explicable on the basis that the latter condition is simply a similar periaortitis involving the thoracic aorta [17]. Beside the presence in the inflammatory mass of predominantly B cells, $\mathrm{T}$ cells and especially T-helper (CD4+) cells were commonly observed. Other inflammatory leukocytes such as plasma cells, polynuclear cells and macrophages were also present. Once entrapped in the atherosclerotic plaque, the macrophages become lipid-laden foam cells and secrete ILs and chemokines that cause cell proliferation (e.g. lymphoctes and fibroblasts). Interleukin (IL)-2 and IL-4 are important in T- and B-cell differentiation and proliferation and the IL-2 receptor is upregulated during inflammation and activation states. Furthermore, consistent with the mRNA results, immunohistochemistry revealed IL-2 receptor- $\alpha$ protein expression in tissue with $\mathrm{CP}$, but not in the non-disease part of the aorta [12]. Studies with appropriate antibodies to IgG and IgM locate these immunoglobulins to areas within the atheromatous plaques where the oil-red $\mathrm{O}$ technique shows the presence of ceroid. The dominant immunoglobulin is IgG. Ceroid-laden macrophages have been identified in the adventitia and nearby lymph nodes in CP. This con- firms the view that $\mathrm{CP}$ is an active, progressive, local immune response associated with advanced ATS.

In a recent study, Moroni et al. [18] found that patients with CP had a significantly increased number of circulating endothelial cells (CECs) in comparison to healthy subjects, patients with diffuse ATS, and patients with renal insufficiency of variable degree and etiology. These data indicate that endothelial injury, as part of immunemediated inflammatory vascular damage, may play a role in the pathogenesis of CP. As far as circulating endothelial phenotype is concerned, the authors found that almost all CECs in patients with CP are microvascular in origin, as defined by the marker CD36. This finding could further suggest that vascular injury in $\mathrm{CP}$ takes its origin from vasa vasorum rather than the aortic layers. In addition, some CECs in patients with $\mathrm{CP}$ had an activated phenotype, as evidenced by the surface expression of E-selectin. Moreover, they found increased plasma concentrations of IL- 6 , an important cytokine with a crucial role as an inflammatory mediator and modulating fibroblast properties.

\section{The Autoimmune Hypothesis}

The association of CP with other autoimmune disorders, as well as the presence of serologic markers of immune activation in a small number of patients, suggests that $\mathrm{CP}$ could be an immune-mediated disorder with marked vascular inflammatory traits. The concept of a systemic disease is also supported by similarities to systemic large-vessel vasculitides. A recent case-control study that compared inflammatory and non-IAAAs showed that IAAAs are more frequently associated with systemic autoimmune diseases [19]. Likewise, in a recent study, Vaglio et al. [20] found that $44 \%$ of CP was associated with other autoimmune conditions, namely ANCApositive renal disease and autoimmune thyroiditis. Finally, various case reports have described an association between $\mathrm{CP}$ and numerous autoimmune diseases including well-defined small- and medium-vessel vasculitis, and fibroinflammatory disorders affecting other organs such as sclerosing cholangitis, mediastinal fibrosis, Riedel's and chronic autoimmune thyroiditis, most of which are thought to have an autoimmune pathogenesis [reviewed in 21]. However, to confirm that vascular uptake in $\mathrm{CP}$ is specific (i.e., due to inflammation of the vessel wall), Salvarani et al. [22] used ${ }^{18} \mathrm{~F}$-fluorodeoxyglucose-positron emission tomography (FDG-PET) to evaluate the presence and extent of large-vessel inflammation in patients 
with CP. The high-grade FDG vascular uptake and the concomitant inflammatory features found in patients with $\mathrm{CP}$ suggested that this vascular involvement may represent active vasculitis.

\section{Influence of Genetics}

It is tempting to speculate that genetic factors are also involved in the presumed relationship between ATS and CP. Indeed, aortic abdominal aneurysm shows a strong familial predisposition. Thus a genetic risk determinant mapped to the HLA-DR molecule, and in particular to the HLA-DRB1 locus and the alleles $\mathrm{B} 1{ }^{*} 15$ and $\mathrm{B} 1^{*} 0404$, suggests a role for genetic risk factors in IAAA [23]. Of interest, substitution of a glutamine for a negatively charged aspartic acid at the entrance to pocket 4 significantly changes the binding of the pocket and therefore changes antigen selection. This suggests a critical role for antigen binding in the origin of the disease, and its distinct location on the HLA-DR molecule suggests disease specificity, compared with giant cell arteritis [19]. On the other hand, certain genetic polymorphisms could predispose to more pronounced inflammatory response and the occurrence of CP. Recently, a link between myoendothelial communication via gap junctions and inflammatory cytokines has been recognized. In addition, a genetic polymorphism of a gap junctional protein has been shown a marker for atherosclerotic plaque development. Analogous genetic polymorphisms could be operative in the development of RPF and/or aneurysm formation, i.e. with regard to MMPs.

\section{Conclusion}

Although the pathogenetic role of atherosclerotic-related processes for the development of CP is an interesting issue that relates to active and ongoing research in the area of ATS and inflammation, several questions remain unanswered. Since only a small percentage of patients with ATS appear to develop clinical disease (only $5 \%$ of atherosclerotic aneurysms are inflammatory), there may be other factors such as intrinsic immune responsiveness, HLA genotype, antigen processing and localization of antigen, important in the expression of clinical disease. Although autoantibodies to ceroid have been demonstrated in patients with clinical and subclinical CP, they were also seen in a high percentage of elderly patients without demonstrable disease and in patients with ATS but without clinical disease, indicating that antigenic exposure is common whereas clinical disease is rare. This may reflect the heterogeneous response of a population to an antigenic challenge, or it may represent an epiphenomenon seen in advanced ATS with no pathophysiological significance. On the other hand, the described association with systemic lupus erythematosus and other connective tissue disease supports the immunologic theories of CP, at least of some forms of CP. Moreover, the beneficial effect of corticosteroid treatment and sporadic reports of benefit from immunosuppressive agents such as azathioprine, cyclosporine or mycophenolate mofetil also fit the hypothesis that $\mathrm{CP}$ represents an autoallergic reaction to components of atheromatous plaques. Further studies to better characterize the above-mentioned factors and the molecular and cellular mechanisms at play in the pathogenesis of $\mathrm{CP}$ are required.

\section{References}

1 Parums DV: The spectrum of chronic periaortitis. Histopathology 1984;16:589-590.

-2 Vaglio A, Salvarani C, Buzio C: Retroperitoneal fibrosis. Lancet 2006;367:241-251.

-3 Mitchinson MJ: The pathology of idiopathic retroperitoneal fibrosis. J Clin Pathol 1970; 23:681-689.

4 Libby P: Inflammation in atherosclerosis. Nature 2002;420:868-874.

5 Ter Maaten JC, Smit AJ, Gans ROB: Idiopathic retroperitoneal fibrosis: idiopathic or secondary to atherosclerosis? Neth J Med 2000;56:35-37.

6 Parums DV: The spectrum of chronic periaortitis. Histopathology 1990;16:423-431.

\footnotetext{
7 Martina FB, Nuech R, Gasser TC: Retroperitoneal fibrosis and chronic periaortitis: a new hypothesis. Eur Urol 1993;23:371-374.

8 Mitchinson MJ: Chronic periaortitis and periarteritis. Histopathology 1984;8:589600.

$\checkmark 9$ Bitisch M, Nørgaard HH, Røder O, Schroeder TV, Lorentzen JE: Inflammatory aortic aneurysms: regression of fibrosis after aneurysm surgery. Eur J Vasc Endovasc Surg 1977;13:371-374.
}

10 O'Brien KD, McDonald TO, Chait A, Allen $\mathrm{MD}$, Alpers CE: Neovascular expression of E-selectin, intercellular adhesion molecule-1, and vascular cell adhesion molecule-1 in human atherosclerosis and their relation to intimal leukocyte content. Circulation 1996;93:672-682.

11 Wood KM, Cadogan MD, Ramshaw AL, Parums DV: The distribution of adhesion molecules in human atherosclerosis. Histopathology 1993;22:437-444.

12 Ramshaw AL, Roskell DE, Parums DV: Cytokine gene expression in aortic adventitial inflammation associated with advanced atherosclerosis (chronic periaortitis). J Clin Pathol 1994;47:721-727. 
13 Freestone T, Turner RJ, Coady A, Higman DJ, Greenhalgh RM, Powell JT: Inflammation and matrix metalloproteinases in the enlarging abdominal aortic aneurysm. Arterioscler Thromb Vasc Biol 1995;15:11451151.

-14 Newman KM, Jean-Claude J, Li H, Ramey WG, Tilson MD: Cytokines that activate proteolysis are increased in abdominal aortic aneurysms. Circulation 1994;90:224227.

-15 Longo GM, Xiong W, Greiner TC, Zhao Y, Fiotti N, Baxter BT: Matrix metalloproteinases 2 and 9 work in concert to produce aortic aneurysms. J Clin Invest 2002;110:625632.

16 Petersen E, Gineitis A, Wagberg F, Angquist KA: Activity of matrix metalloproteinase-2 and -9 in abdominal aortic aneurysms. Relation to size and rupture. Eur J Vasc Endovasc Surg 2000;20:457-461.
7 Parums DV, Chadwick DR, Mitchinson MJ: The localisation of immunoglobulin in chronic periaortitis. Atherosclerosis 1986; 61:117-123.

18 Moroni G, Del Papa N, Moronetti LM, Vitali C, Maglione W, Comina DP, Urgnani F, Sandri S, Ponticelli C, Cortelezzi A: Increased levels of circulating endothelial cells in chronic periaortitis as a marker of active disease. Kidney Int 2005;68:562-568.

19 Haug ES, Skomsvoll JF, Jacobsen G, Halvorsen TB, Saether OD, Myhre HO: Inflammatory aortic aneurysm is associated with increased incidence of autoimmune disease. J Vasc Surg 2003;38:492-497.
20 Vaglio A, Corradi D, Manenti L, Ferretti S, Garini G, Buzio C: Evidence of autoimmunity in chronic periaortitis: a prospective study. Am J Med 2003;114:454-462.

21 Meier P, Rotman S, Blanc E, Burnier M: Retroperitoneal fibrosis, a true inflammatory disease: new insights in an old entity; in Pitzer JA (eds.): Progress in Inflammation Research. New York, Nova Publishers, 2005, pp 3-45.

22 Salvarani C, Pipitone N, Versari A, Vaglio A, Serafini D, Bajocchi G, Salvo D, Buzio C, Greco P, Boiardi L: Positron emission tomography: evaluation of chronic periaortitis. Arthritis Rheum 2005;53:298-303.

$>23$ Rasmussen TE, Hallett JW Jr, Metzger RL, Richardson DM, Harmsen WS, Goronzy JJ, Weyand CM: Genetic risk factors in inflammatory abdominal aortic aneurysms: polymorphic residue 70 in the HLA-DR B1 gene as a key genetic element. J Vasc Surg 1997;25: 356-364. 